EPJ Web of Conferences 110,01031 (2016)

DOI: $10.1051 /$ epjconf/201611001031

(C) Owned by the authors, published by EDP Sciences, 2016

\title{
PROTECTIVE EFFECTIVENESS OF POROUS SHIELDS UNDER THE INFLUENCE OF HIGH-SPEED IMPACT LOADING
}

\author{
E.N. Kramshonkov ${ }^{1,}$, , A.V. Krainov ${ }^{1}$, P.V. Shorohov ${ }^{1}$ \\ ${ }^{1}$ National Research Tomsk Polytechnic University, 634050 Tomsk, Russia
}

\begin{abstract}
The results of numerical simulations of a compact steel impactor with the aluminum porous shields under high-speed shock loading are presented. The porosity of barrier varies in wide range provided that its mass stays the same, but the impactor has always equal (identical) mass. Here presented the final assessment of the barrier perforation speed depending on its porosity and initial shock speed. The range of initial impact speed varies from 1 to $10 \mathrm{~km} / \mathrm{s}$. Physical phenomena such as: destruction, melting, vaporization of a interacting objects are taken into account. The analysis of a shield porosity estimation disclosed that the protection effectiveness of porous shield reveals at the initial impact speed grater then $1.5 \mathrm{~km} / \mathrm{s}$, and it increases when initial impact speed growth.
\end{abstract}

\section{Introduction}

The space vehicles protection from high-velocity cosmic dangerous objects (CDO) is of the great practical importance. CDO possess technogeneous and naturally occurring origin. As a rule CDO are: comets particles, cosmic rubbish, space powder, micrometeorite. In general, the natural origin CDO subdivided on kinds: "iron", "stony" and "aqueous". Among them iron cosmic particles - are the most dangerous. On the other side, high-velocity iron CDO of the technogeneous origin pose a great threat to the spacecrafts to be affected to high-speed shock. Therefore the compact steel impactor as the striker was considered. The metal alloys utilization as space vehicles structural elements - is a traditional approach in the spacecrafts manufacturing. The porous materials usage is of a great interest so far as permits the mass of protective construction reduce. In this paper the influence of barrier porosity and initial impact speed on barrier perforation speed is considered. It is shown that a porous barrier effectiveness emerges under the high speed interaction. The low-range of initial impact speeds emerges effectiveness of monolithic barriers [1].

A mathematical description of the behavior of the interacting bodies is performed using an elastoplastic model of continuum mechanics taking into account the variation in the porosity of the deformed materials and with a variable yield limit [2]. The porous continuum as the two-componential, homogeneous and isotropic material that is composed of a monolithic matrix medium and a spherical porous is represented. The density of a porous material specifies by means of the parameter named porosity $\alpha=\rho_{m} / \rho,\left(\rho_{m}\right.$-density of a matrix material). The relative volume of a medium porous by means of formula $\eta=(\alpha-1) / \alpha$ is expressed, and numerical values of $\eta$ in percentage are signified. Various states of aggregation by means of an interpolation wide-range equations of state describing the solid. liquid and gaseous phases are modeled [3]. This equation in a wide range of pressure and temperatures describes thermodynamic behavior of materials. It means that, the thermodynamic substance condition in both condensed, and a gaseous phase is characterized. That is absolutely essential for a numerical modeling of the hypervelocity impact phenomena.

The elastoplastic model of continuum includes two strength property: yield limit and shear modulus. It is supposed, that yield limit and shear modulus depends on the porosity pressure and level of internal energy in relation to energy of fusion. All three factors independently from each other in various directions and with various intensity operates. For example, the yield limit increases in accordance with the linearly varying law from the minimum value to the maximum only when pressure increments. However increment value of internal energy up to fusion energy reduces the yield limit till zero. In this case, the elastoplastic model into an ideal fluid model is transformed.

The model for failure calculations includes a joint consideration of two failure mechanisms: shear and spall [2,4]. The growth and accumulation of microdamage were taken into account by correcting the characteristic parameters of the medium modeled. Though one of failure mechanisms is dominant, their combinations often is received. Therefore to get the most adequate description of an failure process it is necessary that two failure mechanisms of the medium should be realized. As a prediction model of a shear failure a specific work of shear plastic deformations is used. As a prediction model of a spall failure a relative volume of a medium porous is used. A shear failure is preliminary, it does not pose the fragmentation of interacting bodies [5-10]. The material after failure is modeled by a loose medium (sand or dust), which is not capable of

\footnotetext{
${ }^{\mathrm{a}}$ Corresponding author: lux_veritatis@mail.ru
} 


\section{EPJ Web of Conferences}

resisting tension but resist compressive loads and shear deformation in compression. An additional technique component is the algorithm of disintegration of interacting bodies, that is to say continuity disruption into separate fragments.

The numerical modeling technique in Eulerian spatial variables used in the present work is based on a two-step scheme of the predictor-corrector type and the method of discrete markers. This technique allows calculations of large satins of interacting bodies and has no restrictions on impact velocity and geometry of interacting bodies [2].

\section{Problem statement, the initial data and analysis results}

Physically the problem is formulated as a high-speed shock on a target by a compact cylindrical impactor. Physical statement of a problem is illustrated on figure 1 . The shock velocity $V_{0}$ is directed along an axis of the impactor and at right angle to a barrier. The problem at the axisymmetric statement is considered. The compact impactor is the steel cylinder (the diameter $d=10 \mathrm{~mm}$ and the height $h=10 \mathrm{~mm}$ ). Barriers are aluminum plates of identical specific weight (the thickness of barriers vary from $30 \mathrm{~mm}$ to 150 $\mathrm{mm}$ ), and initial porosity is various. The relative volume of a porous of barriers $\eta$ varies from $0 \%$ to $80 \%$ (see figure 1 ).

As an example two variants of a problem about high-speed compact impactor penetration on a monolithic and porous barrier are considered. The initial speed of shock is $8 \mathrm{~km} / \mathrm{s}$. The first stage of penetration process, while impactor still slightly deformed is presented on figure 2. The arrows on the upper half-plane show the field of mass speeds and maximum length of an arrow corresponds to the maximum value of mass speed $V \max$. Numerical values of $V \max$ is shown on explanatory notes to figure 2. Here numerical values $P \max$ is the maximum pressure, $V k$ is the speeds of contact border (impactor penetration speed).

On explanatory notes to figure 2 various conditions of the failed fragments of impactor and barrier are labeled. Under the figure a brief explanation of a various conditions is given. The SHEAR - a material is failed as shift, the SPALL - a material is failed as a separation, MELTING - internal energy more than energy of fusion, $P$ GAS - internal energy of a material more than energy of sublimation, GAS - the substance is evaporated. Elimination of the evaporated fragments allows simplifying the analysis of a problem [11-22].

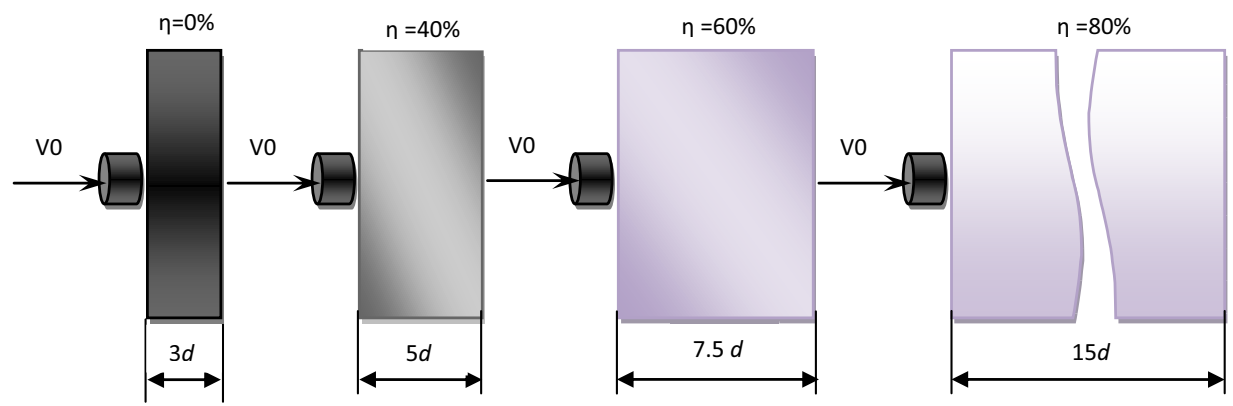

Figure 1. An initial configuration of interacting objects.

The areas of light grey color indicate material fusion and the dark areas correspond to a material condition, while its internal energy more than sublimation energy. However, this fact does not mean that the material in the dark area belong to gaseous phase on account of after unloading its internal energy will be decreased. For the phase transition into the gaseous condition it is necessary, that after unloading to zero pressure internal energy of a material remained more than sublimation energy. By the time presented on figure 2 spall and shear failure yet are not observed. It is important to notice, that according to the proposed failure model fusion can be considered as one of the failure mechanism. After material is fused its shear modulus, yield strength and tensile strength receive zero values.
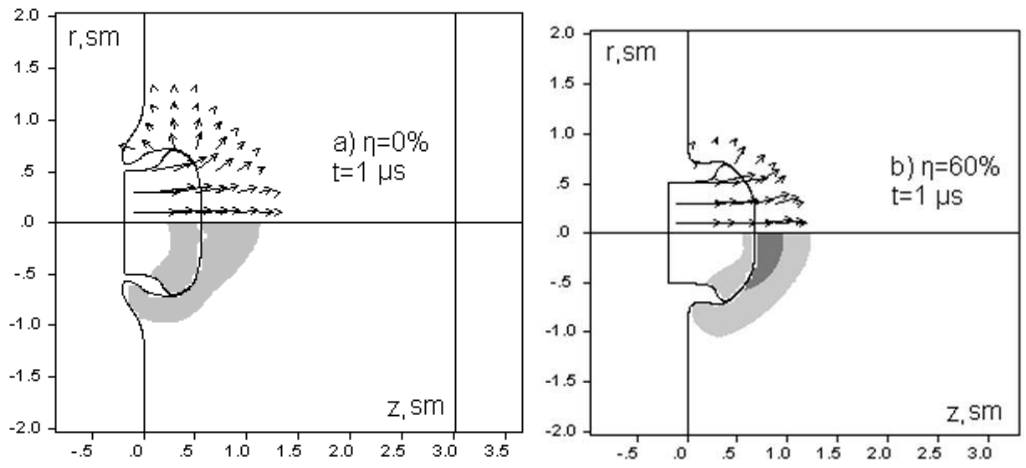


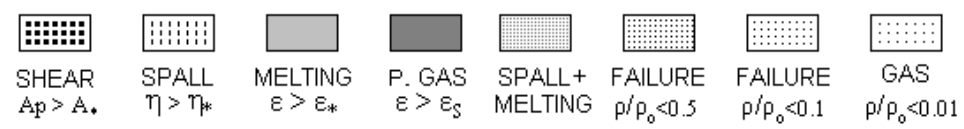

Figure 2. An initial stage of penetration process, velocity of impact is $8 \mathrm{~km} / \mathrm{s}: a-V \max =7.9 \mathrm{~km} / \mathrm{s}, P \max =59 \mathrm{GPa}, V k=4.9 \mathrm{~km} / \mathrm{s} ; b-V \max =$ $8.0 \mathrm{~km} / \mathrm{s}, P \max =41 \mathrm{GPa}, V k=6.3 \mathrm{~km} / \mathrm{s}$.

The penetration mechanism of both shock variants according to the impactor spreading on a crater surface occurs. This process can be separated into three stages. The first stage represented on figure 2, the wave-like behavior, significant deformation and complete barrier fusion within the interaction area is characterized. Then there comes the second stage of the steady state period of penetration, as the result the impactor material on a bottom of crater arrives and its material on sidewall spreads. The period of time of the second stage is of short duration, while the impactor is compact. A barrier stationary region of compressed material comparatively the contact surface (crater bottom), separating a zone of fusion from unloaded part of a barrier is formed. When this area appears on a free surface of a barrier, the third stage (the final stage) comes, it terminal perforation-speed of the barrier determines.

The mass speed $V z$, the pressure $P$ and the temperature $T$ dependencies on a symmetry axis coordinate on figures 3-5 are illustrated. The figures 3-5 the initial stage of penetration process on a porous and a solid barriers is shown. The penetration speed of the impactor on a porous barrier is of long duration then on a solid barrier. High values of mass speed, pressure and temperature take place in a narrow region of the porous barrier adjacent to a contact surface. There is a in this area of a barrier and it becomes solid. This process a temperature rise on a cavity pockets collapse zone is accompanied (see figure $5, b$ ). Substantial temperature increase of a porous barrier on a region of contact border indicates, that extra work of deformation occurred to cavity pockets collapse process transfers into internal energy of a barrier compression zone, and kinetic momentum on the breach region does not increase.
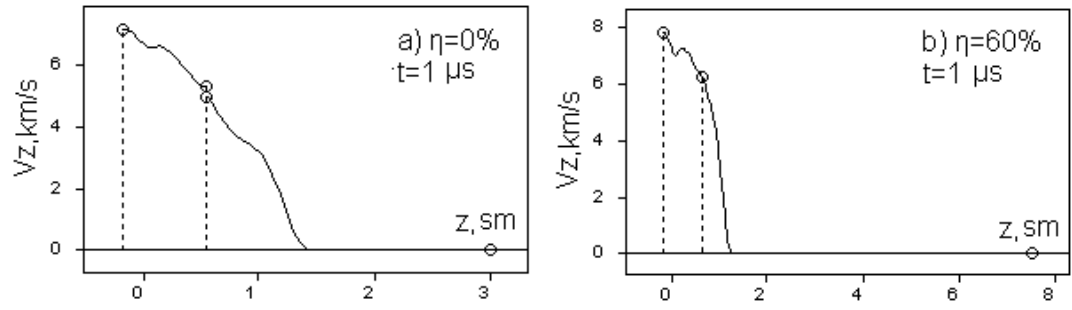

Figure 3. Dependence of mass speed on a symmetry axis coordinate.
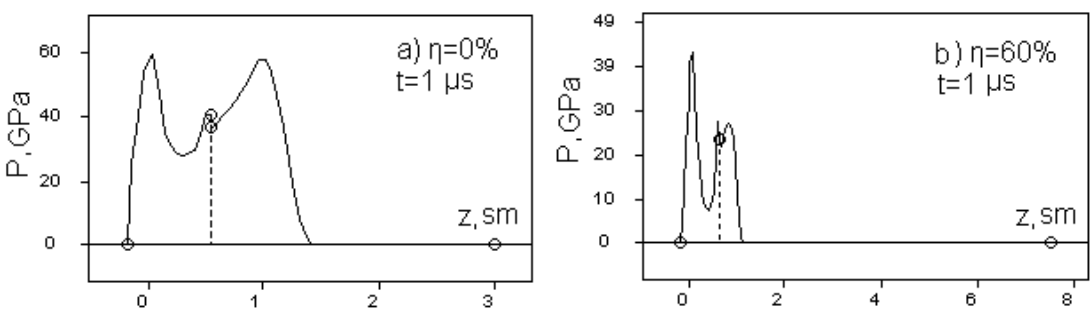

Figure 4. Dependence of pressure on a symmetry axis coordinate.
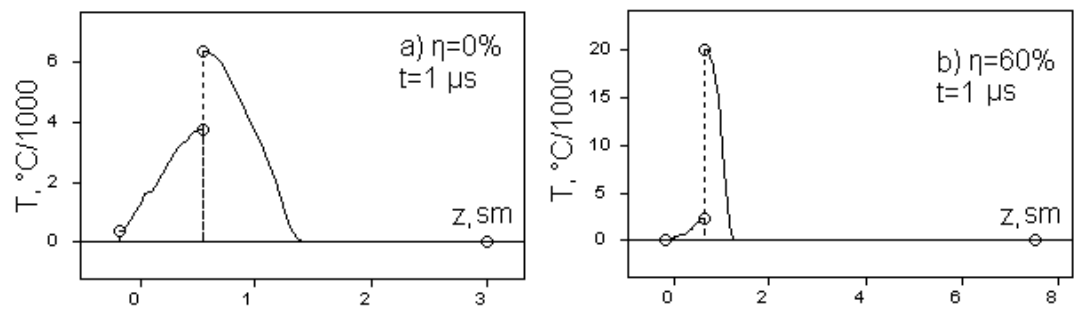

Figure 5. Dependence of temperature on a symmetry axis coordinate.

Later stages of penetration by the time of $t=10 \mu \mathrm{s}$ are presented on figure 6 . The bottom half-plane (see figure 6,a) shows that the spall failure mechanism takes place, the relative volume of a porous medium increased up to the value above $50 \%$. A relative density of the peripheral areas of the impactor and the regions of opposite emission of a barrier material has decreased up to the value $\rho / \rho_{0}=0.1$, i.e. the density of sprayed material has decreased in 10 times in comparison to its initial density. 


\section{EPJ Web of Conferences}

There are not material dispersion effects by the time th the case of impact on porous barrier (see figure 6, b). An opposite emission of the crater peripheral regions is typical of solid barrier (see figure 6,a). Here barrier material up to the lowest values is sprayed and fragmented. As for a porous barrier (see figure $6, b$ ) the similar effects are absent.

The stage of impactor penetration process on a solid barrier (see figure $6, a$ ) when the speeds of interacting bodies equals to each other over the central region is shown. The mass speed dependence on symmetry axis coordinate confirms that fact (see figure $7, a$ ).

The stage of a penetration process of a porous barrier, when the shock wave did not appear on a free surface is represented on figure $6, b$. The region of high mass speed is enough distant from a free surface of a barrier, and increase of free surface speed is not begun. By that moment striker penetration velocity on a porous barrier almost equal to penetration velocity on a solid barrier. It is shown on figure 6, where numerical values of speed of contact surface $V k$ are presented. The velocity dependencies on figure 7 also confirm that fact.

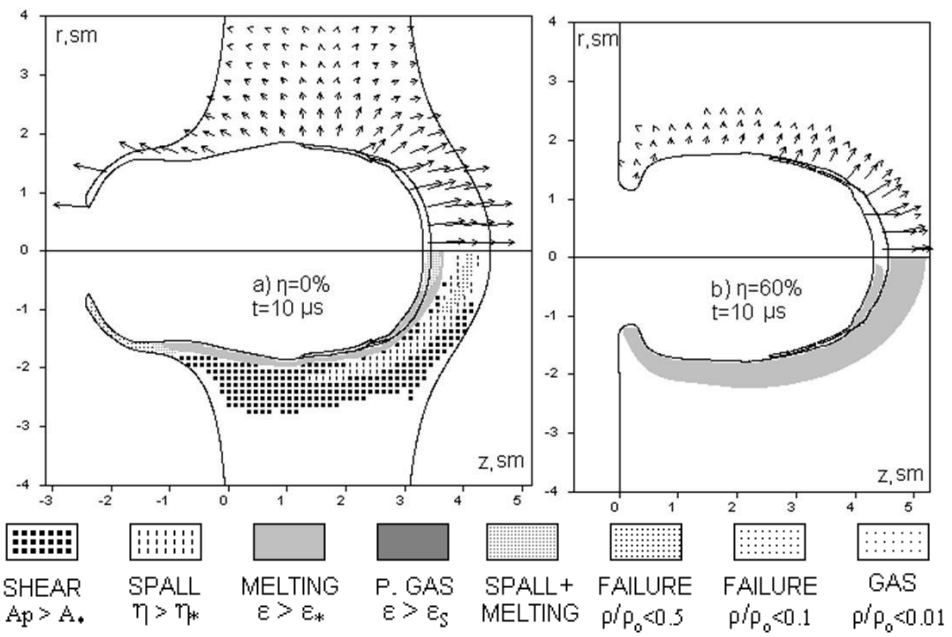

Figure 6. The impactor spreading on a crater surface, velocity of impact is $8 \mathrm{~km} / \mathrm{s}: a-V \max =2.7 \mathrm{~km} / \mathrm{s}, P \max =2 \mathrm{GPa}, V k=2.6 \mathrm{~km} / \mathrm{s} ; b-$ $V \max =2.9 \mathrm{~km} / \mathrm{s}, P \max =5.8 \mathrm{GPa}, V k=2.7 \mathrm{~km} / \mathrm{s}$.

The penetration process kinematics is characterized on figure 8 , where time dependences of the speed of contact surface $V k$ and speed of free surface $V t$ is presented. The solid barrier speed alignment of $V k$ and $V t$ occurs by the moment $t=14$. As for the porous barrier $V k$ becomes equal to $V t$ by the moment $t=40 \mu \mathrm{s}$. During that period of time contact surface speed of porous barrier decreases by the greater value then contact surface speed of solid barrier (see figure $8, a$ ). When the speeds $V k$ and $V t$ become equal to each other their further decrease occurs no more $200 \mathrm{~km} / \mathrm{s}$, i.e. it is possible to consider an value of average speed as the perforation velocity.
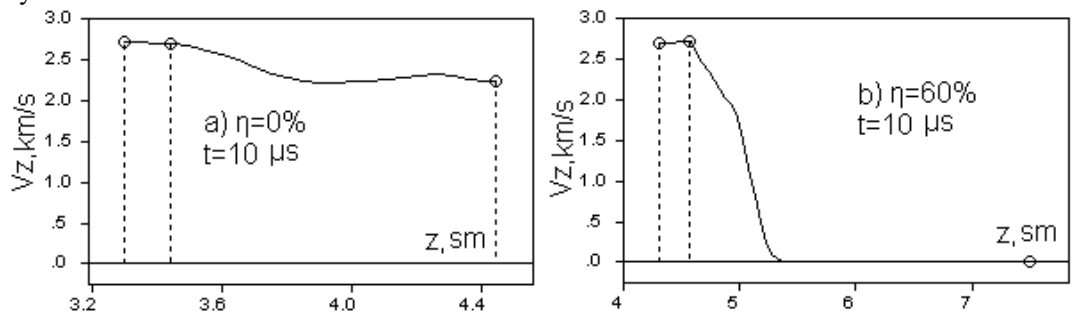

Figure 7. Dependence of mass speed on a symmetry axis coordinate. 


\section{Thermophysical Basis of Energy Technologies 2015}
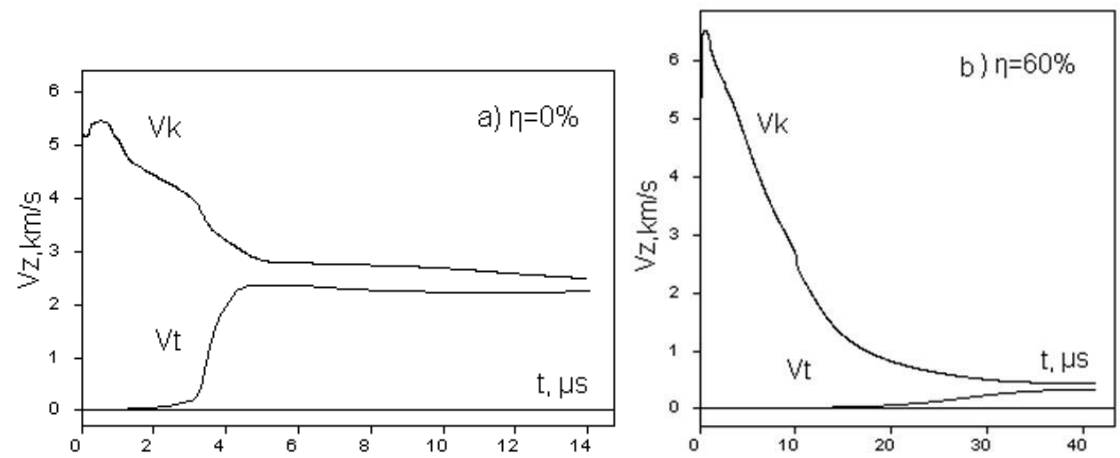

Figure 8. Dependence of velocity of contact surface $V k$ and velocity of free surface $V t$ on time.

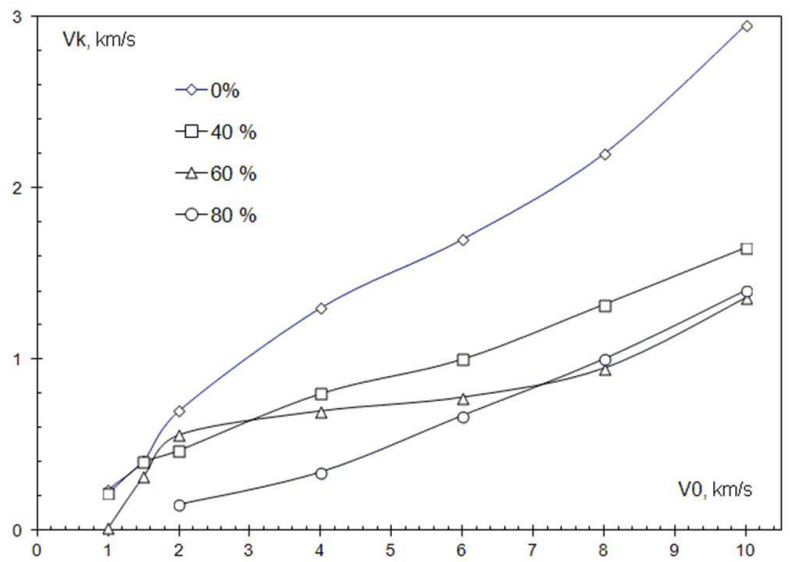

Figure 9. Dependence of perforation speed on initial velocity of impact.

Therefore time factor is a principal cause of a greater speed decrease of an impactor penetration, i.e. the greater duration of a time interval of the speed alignment $V k$ and $V t$. It is important to mention, that during an initial stage penetration process contact surface speed of porous barrier is greater then contact surface speed of solid barrier, and when $t>10 \mu$ s invert correlation of values of contact surface speed is observed.

To specify character of dependencies barrier porosity and initial velocity of impact on the terminal perforation speed, a calculations series of impactor penetration on barriers of various initial porosity is realized. The range of initial shock speed varies from 1 to $10 \mathrm{~km} / \mathrm{s}$. Dependence of a perforation speed on an initial speed of impact is plotted on figure 9. Hire percentages indicate the relative volume of a medium porous of a barrier. An advantage of a porous barrier over solid barrier over all investigated range of initial shock velocity is total. When initial shock velocity increase, the effectiveness of a porous barrier growth too. when initial shock velocity lower than $1.5 \mathrm{~km} / \mathrm{s}$, impactor barely deformed and the effectiveness of a porous barrier is insignificant.

The work was realized within the research state assignment "Science” №13.1339.2014/K (Code of Federal Target Scientific and Technical Program 2.1410.2014).

\section{References}

1. V.G. Trushkov, E.N. Kramshonkov, E.A. Jagnitsina, J. News of high schools. Physics, 194 (2007)

2. V.G. Trushkov, Numerical investigation of high-speed interaction objects of large deformation medium in the Euler space variables (2007)

3. A.V. Zhukov, J. Deformable solid mechanics, 70 (1987)

4. V.F. Tolkachyov, V.G. Trushkov, J. Chem. Phys., 2, 170 (1993)

5. L.V. Efremova, A.I. Korneev, V. G. Trushkov, J. Physics of combustion and explosion, 2, 110 (1987)

6. C.V. Razorenov, G.I. Kanel, E.N. Kramshonkov, K. Baumung, J. Physics of combustion and explosion, 5, 119 (2002)

7. A. V. Kraynov, G. V. Kuznetsov, EPJ Web of Conferences, 76, 01023 (2014)

8. A.V. Kraynov, E.N. Pashkov, A.V. Ponomarev, Advanced Materials Research, 1040 (2014)

9. G.V. Kuznetsov, A.V. Krainov, J. Appl. Mech. Tech. Phys., 5, 851 (2001)

10. G.V. Kuznetsov, A.V. Krainov, J. High Temperature, 2, 252 (2003) 


\section{EPJ Web of Conferences}

11. M. Yu. Orlov, Yu. N. Orlova, V. F. Tolkachev, Investigation of the behavior of ice and limestone under explosive loading, (XXX International Conference on Interaction of Intense Energy Fluxes with Matter March Elbrus, Kabardino-Balkaria , 2015)

12. M. Matbou Riahi, D. Marceau, C. Laforte, J. Perron, Cold Reg. Sci. Technol., 65, 191 (2011)

13. M. Kermani, Masoud Farzaneh, Robert Gagnon, Cold Reg. Sci. Technol., 53, 162 (2008)

14. H. Ada, R. Liamazares, K. Hoyland, E. Kim, Cold Reg. Sci. Technol., 65, 298 (2011)

15. M. Rudolph, M. Manga, Fracture penetration in planetary ice shells, Icarus 199, 536 (2009)

16. J. Dong, Z. Li, P. Lu, Q. Jia, G. Wang, G. Li, Cold Reg. Sci. Technol., 71, 34 (2012)

17. M. Yasui, M. Arakawa, Icarus 210, 956 (2010)

18. A. Combescure, Y. Chuzel-Marmot, J. Fabis, Int. J. Solids Struct. 48, 2779 (2011)

19. A.C. Palmer, J.P. Dempsey, D.M. Masterson, Cold Reg. Sci. Technol., 56, 73 (2009)

20. G. Appleby-Thomas, P. Hazell, G. Dahini, Composite Structures, 93, 2619 (2011)

21. H. Park, H. Kim, International Journal of Impact Engineering, 37, 177 (2010)

22. R. Ritch, R. Frederking, M. Johnston, R. Browne, Cold Reg. Sci. Technol., 52, 29 (2008) 